\title{
Impacto de la disfunción sistólica y diastólica en la comunidad
}

Burden of systolic and diastolic ventricular dysfunction in the community: appreciating the scope of the heart failure epidemic. Redfield MM, Jacobsen SJ, Burnett JC Jr, Mahoney DW, Kr De Bailey, Rodeheffer RJ. JAMA.2003;289(2):194-202.

\section{Objetivo}

Determinar en la comunidad la prevalencia de Insuficiencia Cardíaca (IC), de disfunción diastólica (DD) y sistólica (DS) preclínicas y determinar si la DD es predictora de mortalidad global.

\section{Diseño}

Estudio de corte transversal y seguimiento de mortalidad por una mediana de 3,5 (2,9-4,2) años.

\section{Lugar}

Condado de Olmsted, Minnesota, EE.UU.

\section{Pacientes}

Selección aleatoria (de 1997 al 2000) de 4203 residentes $\geq 45$ años de los que participaron 2042 (47\%).

\section{Medición de resultados}

Función sistólica y diastólica mediante ecocardiograma doppler: DD leve (relajación demorada sin aumento de presión de llenado), DD moderada (con moderada elevación de presión de llenado, pseudonormal) y DD severa (muy baja distensibilidad o patrón de llenado restrictivo). Presencia de diagnóstico de IC según la revisión de historias clínicas y validación por criterios de Framingham. Las personas sin diagnóstico de IC pero con DD o DS eran consideradas como preclínicas.

\section{Resultados}

El promedio de edad era de $62,8 \pm 10,6$ años. El análisis de 500 historias clínicas seleccionadas al azar de no-participantes no halló diferencias con los participantes entre los distintos factores de riesgo.La prevalencia de IC validada fue 2,2\% (IC 95\%: 1,6-2,8\%) y $44 \%$ de éstos tenían una Fracción de Eyección (FE) normal $(\geq$ $50 \%)$. La prevalencia de IC aumentó significativamente con la edad
$(8,4 \%$ en $\geq 75$ años) y era ligeramente mayor en hombres.El $20,8 \%$ $(19-22,7 \%)$ de la población tenía DD leve, $6,6 \%(5,5-7,8 \%)$ moderada, y $0,7 \%(0,3-1,1 \%)$ severa. El $5,6 \%(4,5-6,7 \%)$ de la población tenía DD moderada-severa aislada (con FE normal).La severidad de la DD se asoció significativamente a la masa ventricular y al volumen auricular izquierdo. La prevalencia de cualquier DS (FE $\leq 50 \%$ ) fue $6,0 \%(5-7,1 \%)$ y de DS moderada o severa (FE $\leq$ $40 \%) 2,0 \%(1,4-2,5 \%)$.La prevalencia de DD y DS aumenta con la edad pero sólo la DS era mayor en hombres. Aún entre pacientes con DD o DS moderada o severa, más de la mitad eran preclínicas (sin IC reconocida).La prevalencia de DD y DS preclínicas es considerable en especial en población de alto riesgo (tabla). El riesgo relativo* de mortalidad global en el análisis multivariado*, controlando por edad, sexo y FE, fue para DD leve $8,3(3-23,1 ; p<0,001)$ y para DD moderada o severa $10,2(3,3-31 ; p<0,001)$.

Tabla. Prevalencia de DD y DS cada 100 personas en la comunidad (IC95\%)

\begin{tabular}{l|c|c|c|c}
\multirow{2}{*}{ Población } & \multicolumn{2}{|c|}{ DS } & \multicolumn{2}{c}{ DD } \\
\cline { 2 - 5 } General & $\mathrm{FE} \leq 40 \%$ & $\mathrm{FE} \leq 50 \%$ & leve & moderada- severa \\
- Hombres & $1,1(0,7-1,7)$ & $4,9(4,0-6,0)$ & $20,6(18,7-22,6)$ & $6,8(5,6-8,0)$ \\
- Mujeres & $2,0(1,2-3,1)$ & $7,9(6,3-9,8)$ & $22,3(19,5-25,3)$ & $6,2(4,7-8,1)$ \\
\hline & $0,3(0,1-0,8)$ & $2,2(1,4-3,3)$ & $19,1(16,7-21,8)$ & $7,3(5,7-9,1)$ \\
\hline De alto riesgo* & $2,8(1,4-4,9)$ & $10,9(8,0-14,4)$ & $47,6(42,1-53,1)$ & $16,5(12,6-20,9)$ \\
-Hombres & $5,1(2,5-9,2)$ & $16,8(11,9-22,8)$ & $48,7(40,7-56,8)$ & $14,6(9,5-21,0)$ \\
- Mujeres & $0,5(0,0-2,8)$ & $5,0(2,4-9,0)$ & $46,5(38,8-54,3)$ & $18,2(12,7-24,9)$ \\
\hline
\end{tabular}

*Edad $\geq 65$ años + hipertensión arterial (HTA) o enf ermedad coronaria

\section{Conclusiones}

Es prevalente en la comunidad la DS sin IC reconocida. Además, es común la DD (rigurosamente definida por ecocardiograma doppler), a menudo no acompañada por IC reconocida, y asociada a un aumento marcado de la mortalidad global.

Fuente de financiamiento: Public Health Service, Fundaciones Marriot y Mayo, y Miami Heart Research Institute.

\section{Comentario}

Recientes guías de práctica de IC reconocen la eficacia del tratamiento para evitar o demorar la progresión de DS preclínica a $I C^{1}$ y algunos estudios indican que la DD es un factor de riesgo independiente de IC y de muerte cardíaca. ${ }^{2-3}$ Por otro lado la mitad de los pacientes con IC tiene DD aislada (sin baja FE) ${ }^{4-7}$ pero la prevalencia en la comunidad de DS, y especialmente de DD, en relación a la IC era incierta. Este estudio provee la primera estimación de la prevalencia de DD en la comunidad valorada por estrictos criterios doppler. Confirmó que $44 \%$ de los pacientes con IC tenían FE normal, que la DD es común y que la DD aislada es tan común como la DS. La prevalencia de IC se incrementa sustancialmente con la severidad de la DD aunque es frecuente que muchas DD severas no tengan diagnóstico reconocido de IC. Tanto la DD leve como la moderadasevera son predictoras independientes de mortalidad global.La DS también es común y menos de la mitad de los pacientes con FE

\section{Dr. Agustín Ciapponi [ Unidad de Medicina Familiar.Hospital Italiano de Buenos Aires ]}

Referencias

1. Hunt SA, Baker DW, Chin MH, et al. ACC/AHA guidelines for the evaluation and management of chronic heart failure in the adult: executive summary. A report of the American College of Cardiology/American Heart Association Task Force on Practice Guidelines (Committee to revise the 1995 Guidelines for the Evaluation and Management of Heart Failure).J Am Coll Cardiol.2001;38(7):2101-13

2. Aurigemma GP, Gottdiener JS, Shemanski L, Gardin J, Kitzman D. Predictive value of systolic and diastolic function for incident congestive heart failure in the elderly:the cardiovascular health study.J Am Coll Cardiol.2001;37(4):1042-8

3. Bella JN, PalmieriV, Roman MJ, Liu JE, Welty TK, Lee ET, Fabsitz RR, Howard BV, Devereux RB. Mitral ratio of peak early to late diastolic filling velocity as a predictor of mortality in middle-aged and elderly adults:the Strong Heart Study.Circulation.2002;105(16):1928-33.

4. Senni M, Tribouilloy CM, Rodeheffer RJ, et al. Congestive heart failure in the community: a study of all incident cases in Olmsted County, Minnesota, in 1991. Circulation.1998;98:2282-2289. 4. Senni M, Tribouilloy CM, Rodeheffer RJ, et al. Congestive heart failure in the community: a study of all incident cases in Olmsted County, Minnesota, in 1991. Circulation.1998;98:2282-2289.
5. Vasan RS, Larson MG, Benjamin EJ, Evans JC, Reiss CK, Levy D. Congestive heart failure in subjects with normal vs reduced left ventricular ejection fraction. J Am Coll Cardiol. 1999;33:1948-1955

6.Kitzman DW, Gardin JM, Gottdiener JS, et al, for the CHS Research Group. Cardiovascular Health Study. Importance of heart failure with preserved systolic function in patients > or $=65$ years of age.Am J Cardiol.2001;87:413-419.

7.D evereux RB, Roman MJ, Liu JE, et al. Congestive heart failure despite normal left ventricular systolic function in a population-based sample:the Strong Heart Study.Am J Cardiol. 2000;86:1090-1096.

8.Lloyd-Jones DM.The risk of congestive heart failure:sobering lessons from the Framingham Heart Study.Curr Cardiol Rep.2001;3:184-190 